\section{Potential Cost-Effectiveness of US-Guided FNAC in Melanoma Patients as a Primary Procedure and in Follow-Up}

\section{TO THE EDITORS:}

We read with great interest the vivid correspondence between the authors of recent papers in the Annals of Surgical. ${ }^{1-3}$ The discussion focuses on a possible survival benefit for patients treated by sentinel lymph node biopsy (SNLB) procedure compared with observation (OBS) and the potential cost-effectiveness of SNLB in the light of such a supposed survival benefit, based on the third interim results of the prospective Multicenter Selective Lymphadenectomy Trial-1 (MSLT-1). ${ }^{4}$

Interestingly, the discussion also focuses on the nodal relapse rates for both arms of the MSLT-1 trail (Table 1). Strikingly, there seems to be an increase in late relapses in both arms, which might either be the result of selection bias, as follow-up has not yet matured to 10 years in the entire MSLT-1 population and thus might lead to an overestimation of the data, or these continuous late relapses in both arms may indicate a failure rate of completion lymph node dissection (CLND) completeness. Interestingly we did not see any late relapses in our submicrometastases $(<0.1 \mathrm{~mm})$ patients. $^{5-7}$

In light of the lack of survival benefit for the sentinel node (SN) procedure from the point of randomization in the MSLT-1 trial, the cost-effectiveness of the SN procedure as a staging procedure is debatable. Recently a study by Voit et al. demonstrated that presurgical ultrasound (US)-guided fine-needle aspiration cytology (FNAC) has a sensitivity of $65 \%$ compared with surgical SN procedure. ${ }^{8}$ Moreover, the sensitivity of US-guided FNAC increases significantly with increasing SN tumor burden. ${ }^{8}$

Considering that only $15-30 \%$ of all stage I/II melanoma patients are SN positive, $70-85 \%$ are negative but still undergo a SN procedure. ${ }^{4}$ US-guided FNAC has the potential to save $65-80 \%$ of $\mathrm{SN}$-positive patients a $\mathrm{SN}$ staging procedure and to save an estimated $61-92 \%$ of $\mathrm{SN}$-negative patients a surgical procedure, and the accompanying costs.

Here we would like to submit the argument for ultrasound-guided FNAC as a cost-effective alternative scenario to the surgical SN procedure. For the purpose of these calculations, based on the data by Voit et al., we considered that $40 \%$ will undergo an US with FNAC whereas $60 \%$ will have a benign US and will not undergo a FNAC. ${ }^{8}$ Moreover, 50\% will be FNAC positive and 50\% will be FNAC negative. Finally, the negative patients will undergo routine US follow-up (four times a year), with an average of one FNAC.

At our centers in The Netherlands and Germany, an SN procedure and 1 day of hospital stay would cost an average of $€ 1254.83$. Thus, for a scenario of 100 stage I/II melanoma patients, the total cost would be $€ 125,483$. Moreover, these calculations do not take into account the time spent in the operating theatre, which could be used for other patients, and the strain on the waiting list for operations.

For the US-guided FNAC scenario, an average ultrasound exam would cost $€ 58.99$ (without FNAC) and a US-guided FNAC would cost an average of $€ 168.61$ at our centers. Figure 1 shows the flowchart of US-guided-FNAC and the sum of the costs. Total costs of US-guided FNAC as an alternative would be $€ 10,283.80+€ 45,831.40=€ 56,115.20$. This is considerably lower than the $€ 125,483$ for $100 \mathrm{SN}$ procedures, corresponding to a cost reduction of $55 \%$.

In reality the savings will be higher as ultrasound is increasingly used for the follow-up of melanoma patients, even after a negative $\mathrm{SN}$ or after a CLND for a positive SN. At present, these patients undergo in an increasing number of centers an ultrasound exam twice a year for 5-10 years. This would lead to costs up to $2 \times 5 \times 100 \times € 58.99=€ 58,990$ (without any FNAC over the entire follow-up period). Thus the potential saving might even be much higher, approximately $70 \%$.

Thus, US-guided FNAC emerges as an alternative and cost-effective staging procedure compared with surgical SN, with the potential to save up to $€ 69,367.80$ ( $>50 \%)$ at our centers, plus the obvious benefits such as saving patients unnecessary surgery, morbidity, operation theatre time, and reducing the strain on the waiting list for operations. Therefore there is a need to reproduce the results from the study by Voit et al. to establish the value of US-guided FNAC. 
TABLE 1 Nodal metastasis rate and rate of increase according to time for sentinel node (SN) and observation (OBS) arms of the Multicenter Selective Lymphadenectomy Trial-1 (MSLT-1)

\begin{tabular}{|c|c|c|c|c|c|}
\hline & $\mathrm{SN}$ arm & Increase & OBS arm & Increase & Difference \\
\hline 0 years & $15.6 \%$ & $15.6 \%$ & $0 \%$ & & $100 \%$ \\
\hline 3 years & $18.9 \%( \pm 1.4 \%)$ & $3.3 \% 1.1 \% /$ year & $13.6 \%( \pm 1.6 \%)$ & $13.6 \% 4.4 \% /$ year & $5.3 \%$ \\
\hline 5 years & $19.4 \%( \pm 1.4 \%)$ & $0.5 \% 0.25 \% /$ year & $16.1 \%( \pm 1.7 \%)$ & $2.5 \% 1.25 \% /$ year & $3.3 \%$ \\
\hline 7 years & $19.6 \%( \pm 1.5 \%)$ & $0.2 \% 0.1 \% /$ year & $17.0 \%( \pm 1.7 \%)$ & $0.9 \% \quad 0.45 \% /$ year & $2.6 \%$ \\
\hline 10 years & $20.8 \%$ & $1.2 \% 0.4 \% /$ year & $20.5 \%$ & $3.5 \% 1.2 \% /$ year & $0.3 \%$ \\
\hline
\end{tabular}

From: Morton, 6th Biannual International Sentinel Node Society meeting, Sydney 2008

Increase in SN arm, years 5-10: $1.4 \%=0.3 \%$ year

Increase in OBS arm, years 5-10: $2.9 \%=0.6 \% /$ year

FIG. 1 Cost-effectiveness flowchart

of ultrasound-guided FNAC

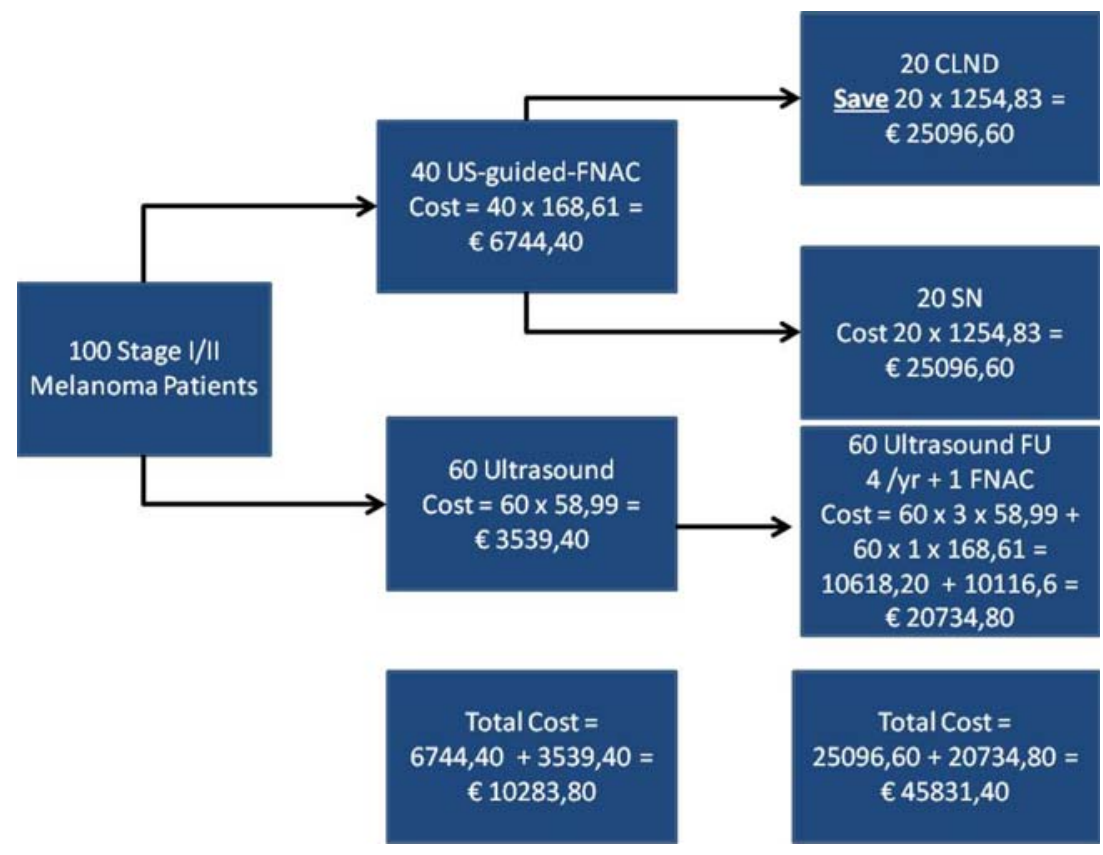

Alexander C. J. van Akkooi, MD, Christiane A. Voit, $\mathbf{P h D}^{2}$, Cornelis Verhoef, $\mathbf{P h D}^{\mathbf{1}}$, and Alexander M. M. Eggermont, $\mathbf{P h D}{ }^{1}$

${ }^{1}$ Department of Surgical Oncology, Erasmus University Medical Center-Daniel den Hoed Cancer Center, Rotterdam, The Netherlands;

${ }^{2}$ Department of Dermatology, Charité, Humboldt University, Berlin, Germany

e-mail: a.vanakkooi@erasmusmc.nl

Published Online: 17 November 2009

(C) The Author(s) 2009. This article is published with open access at Springerlink.com

OPEN ACCESS This article is distributed under the terms of the Creative Commons Attribution Noncommercial License which permits any noncommercial use, distribution, and reproduction in any medium, provided the original author(s) and source are credited.

\section{REFERENCES}

1. Morton RL, Howard K, Thompson JF. The cost-effectiveness of sentinel node biopsy in patients with intermediate thickness primary cutaneous melanoma. Ann Surg Oncol. 2009;16:929-40.

2. Thomas JM. Prognostic false-positivity and cost-effectiveness in sentinel node biopsy in melanoma. Ann Surg Oncol. 2009;16(10): 2961; author reply 2962-3.

3. Thompson JF, Morton DL. In reply: cost-effectiveness of sentinel node biopsy for melanoma. Ann Surg Oncol. 2009;16:2962-3.

4. Morton DL, Thompson JF, Cochran AJ, et al. Sentinel-node biopsy or nodal observation in melanoma. N Engl J Med. 2006;355:1307-17.

5. van Akkooi AC, de Wilt JH, Verhoef C, et al. Clinical relevance of melanoma micrometastases $(<0.1 \mathrm{~mm})$ in sentinel nodes: are these nodes to be considered negative? Ann Oncol. 2006;17:1578-85.

6. van Akkooi AC, Nowecki ZI, Voit C, Schäfer-Hesterberg G, Michej $\mathrm{W}$, de Wilt JH, et al. Sentinel node tumor burden according to the Rotterdam criteria is the most important prognostic factor for survival in melanoma patients: a multicenter study in 388 patients with positive sentinel nodes. Ann Surg. 2008;248(6):949-55.

7. van Akkooi ACJ, Rutkowski P, van der Ploeg IM, et al. Long-term follow-up of patients with minimal sentinel node tumor burden 
$(<0.1 \mathrm{~mm})$ according to the Rotterdam criteria: A study of the EORTC Melanoma Group. J Clin Oncol Suppl. 2009;27:462. Abstract 9005.

8. Voit CA, van Akkooi AC, Schäfer-Hesterberg G, Schoengen A, Schmitz PI, Sterry W, et al. Rotterdam Criteria for sentinel node
(SN) tumor burden and the accuracy of ultrasound (US)-guided fine-needle aspiration cytology (FNAC): can US-guided FNAC replace SN staging in patients with melanoma? J Clin Oncol. 2009;27(30):4994-5000. 